\title{
GPRS-BASED REMOTE SENSING AND CLIMATE CONTROL SYSTEM USING CMOD CPLD
}

\author{
Wael M. El-Medany \\ Department of Communications and Electrical Engineering, \\ Faculty of Engineering, Fayoum University, Egypt \\ Computer Engineering Department, Information Technology College, \\ University of Bahrain, 32038 Bahrain \\ Email: wmelmedany@itc.uob.bh, waelelmedany@gmail.com \\ Webpage: http://people.man.ac.uk/ mbgedwme/ \\ Tel: +973-39764964
}

\begin{abstract}
This paper presents the design and VLSI hardware implementation of a remote sensing system for humidity and temperature in real time. The remote monitoring of the system based on a web design by using GPRS (General Packet Radio Service) network. Since full custom ASIC design takes long time with high cost, programmable logic devices as a programmable ASIC is a better choice for rapid design process and reasonable prices. The design has been described using VHDL (VHSIC Hardware Description Language), and then implemented in hardware using CoolRunner2 CPLD from Xilinx to achieve low cost with rapid prototyping. The design has been simulated and synthesized using Xilinx ISE 6.2i software tools, then test in hardware level using Digilent Spartan 3 starter kit as a hardware tools. The design offers a complete, low cost, powerful and user friendly way of 24 hours real time monitoring system.
\end{abstract}

Keywords: VHDL, GPRS, VLSI, CPLD, Remote Sensing.

\section{INTRODUCTION}

Programmable Logic Devices (PLDs) are extensively used in rapid prototyping and verification of a conceptual design and also used in electronic systems when the mask-production of a custom IC becomes prohibitively expensive due to the small quantity [1]. Many system designs that used to be built in custom silicon VLSI [2] are now implemented in Programmable Logic Devices. This is because of the high cost of building a mask production of a custom VLSI especially for small quantity [3]. With the rapid development of computer technology, the monitored control design of the central airconditioning system is becoming the core of the design for building automation system [4] and [5]. Real-time monitoring provides reliable, timely information of petroleum product's status, important in taking decisions for petroleum production improvement. Evaluation of petroleum production systems is a time consuming and difficult process because it means performing visits to selected petroleum fields to be able to measure and register certain physical, chemical and biological characteristics of the petroleum production areas [6]. Microcontroller has been used for the design of remote sensing and climate control systems [7] and [8]. Field Programmable Gate Array (FPGA) is a better choice than microcontroller for cost effective design, since the use of FPGA will integrate most of the components in one single chip, which in turn reduce the size of PCB [9], [10], and [11]. The disadvantage with FPGA is that it is volatile, once the power is switched off, the desig will be erased. For remote monitoring, GSM has been used by sending SMS messages [12], [13], [14], and [15]. This research introduces a web-based 24-hours real time remote monitoring and climate control system with low cost for building automation and petroleum production systems. The advantages in the introduced system compared to others introduced in [4-15] is the use of GPRS for real time monitoring with low cost compared to the cost of sending SMS in case of GSM MODEM as well as using a 40-pines Coolrunner module "CMOD" with low cost, nonvolatile, and easy for hardware prototyping compared to the use of FPGA. The design has been synthesized and implemented using Xilinx ISE 6.2i. 


\section{SYSTEM ARCHITECTURE}

The system design mainly consists of three main unites: the server, the PC and the remote unit, which in turn consists of four units; the controller that will be implemented in Xilinx CMOD coolrunner-2 CPLD, the sensor circuit, the cooling system, and the GPRS MODEM as shown in Fig. 1. The PC and server are located in the control center and the remote unit is located in the remote land, where humidity and temperature are measured. The three main units are communicated through the internet and the GPRS network. The main function of remote unit is continuously measure the temperature and humidity and compares the measured values with a threshold level, operates the cooling system and sends messages through GPRS network to the control center in case of high temperature or humidity exceeds the threshold level. The main subunit of the remote unit is the controller that has been designed using VHDL, and then implemented on Xilinx Coolrunner CMOD CPLD.
The controller has two components; the first component dealing with humidity measurements; and the second component dealing with the temperature measurements. Each component has its own inputs, the humidity input is coming from the humidity sensor, and the temperature input is coming from the temperature sensor. Both of the humidity and temperature sensors are connected to CPLD through an analog to digital converter (ADC) as shown in Fig. 2. The GPRS is connected to CPLD through RS232, using the standard serial communications port. The communications between GPRS and controller has been achieved by the design of a UART (Universal Asynchronous Receiver Transmitter). The UART has been designed using VHDL, and implemented on the CPLD. On the PC unit a web page has been designed using VB.net, for remotely accessing the system board and sensing the temperature or humidity, as well as sending commands to controller for adjusting the cooling system.

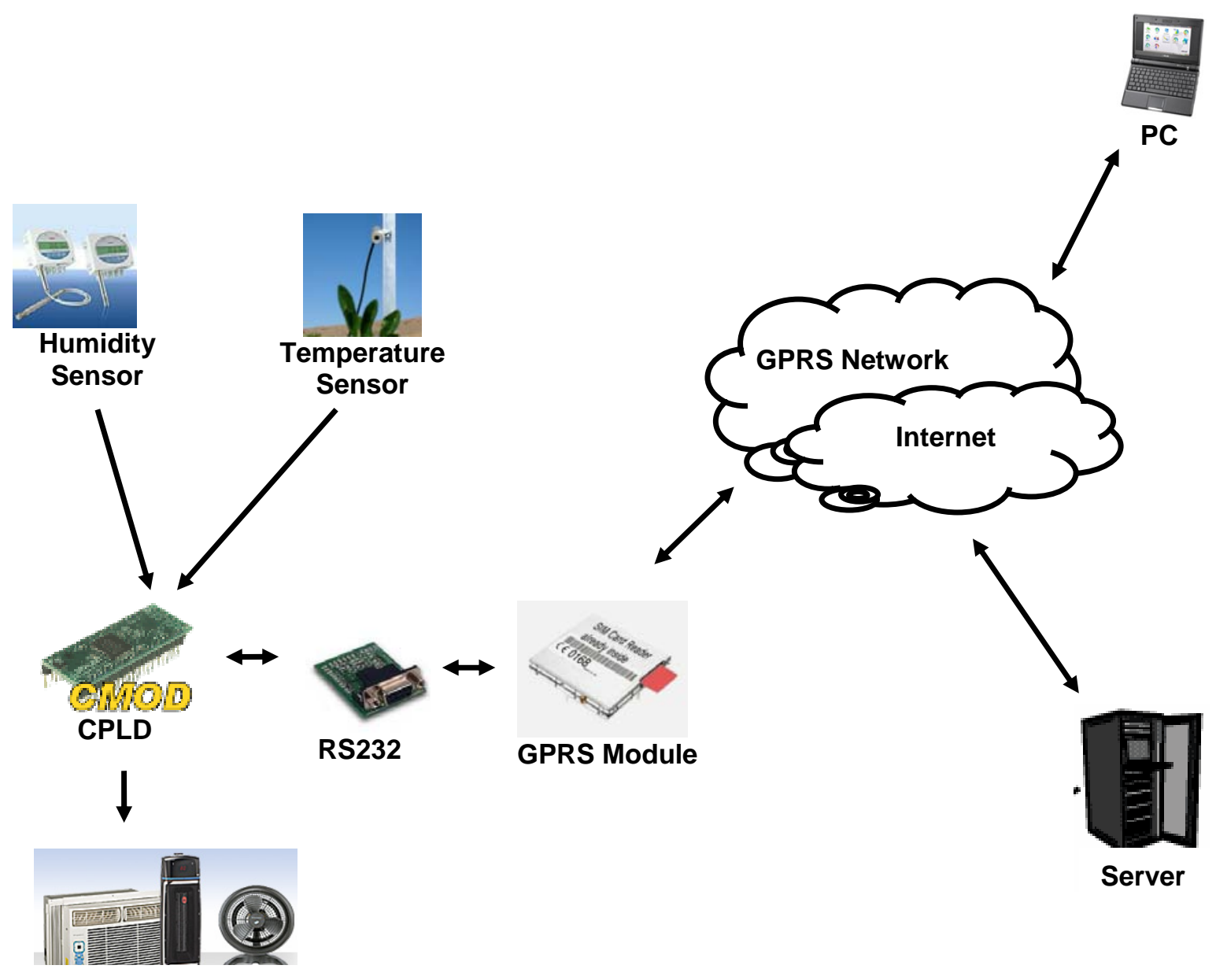

Cooling System

Fig. 1 - System Architecture 


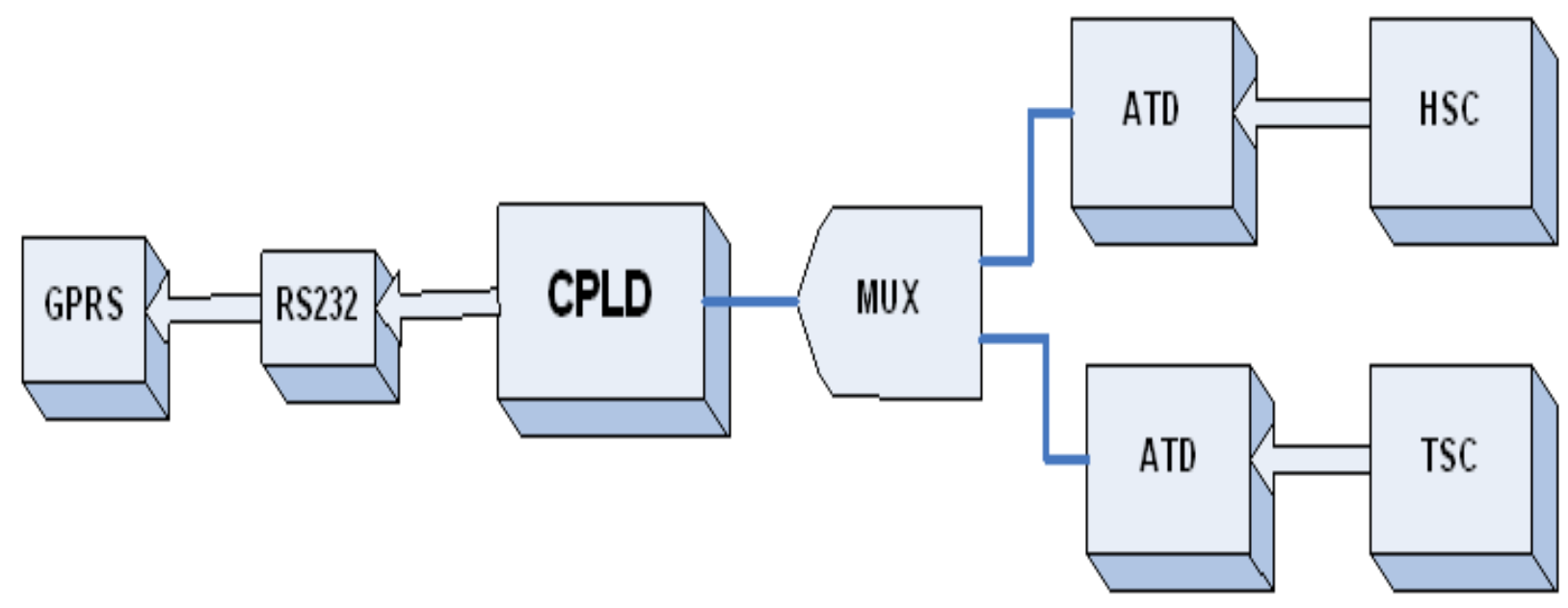

Fig. 2 - Block diagram for the main circuit board unit

\section{STATE MACHINE CHARTS}

This section concerned with hardware design of the controller circuit for the humidity and temperature sensing. Fig. 3 shows the State Machine (SM) Chart for the temperature and humidity control component that has been designed using VHDL and previously mentioned in section 2 . For simplicity we have reduced the number of states to three states; initial state, HS (Humidity Sensing), and TS (Temperature Sensing) as shown in Fig. 3. In the initial state, controller checks for the variable "Sel" which select between temperature and humidity sensing. The HS state checks for high humidity, if it is true, the controller has to adjust the humidity and send message to the control centre, then the control goes back to the initial state. The same scenario happens in TS with temperature sensing. The value "Sel" that select between HS and TS, changes its value sequentially. By this way the controller is continuously monitoring the temperature and humidity status. By comparing the SM chart of fig. 3 with the block diagram of fig. 2, the input "Sel" in SM chart represents the "MUX" block in the block diagram. This multiplexer is used to save the number of used pines from CPLD by multiplexing both of the two reading from the humidity and temperature sensors.

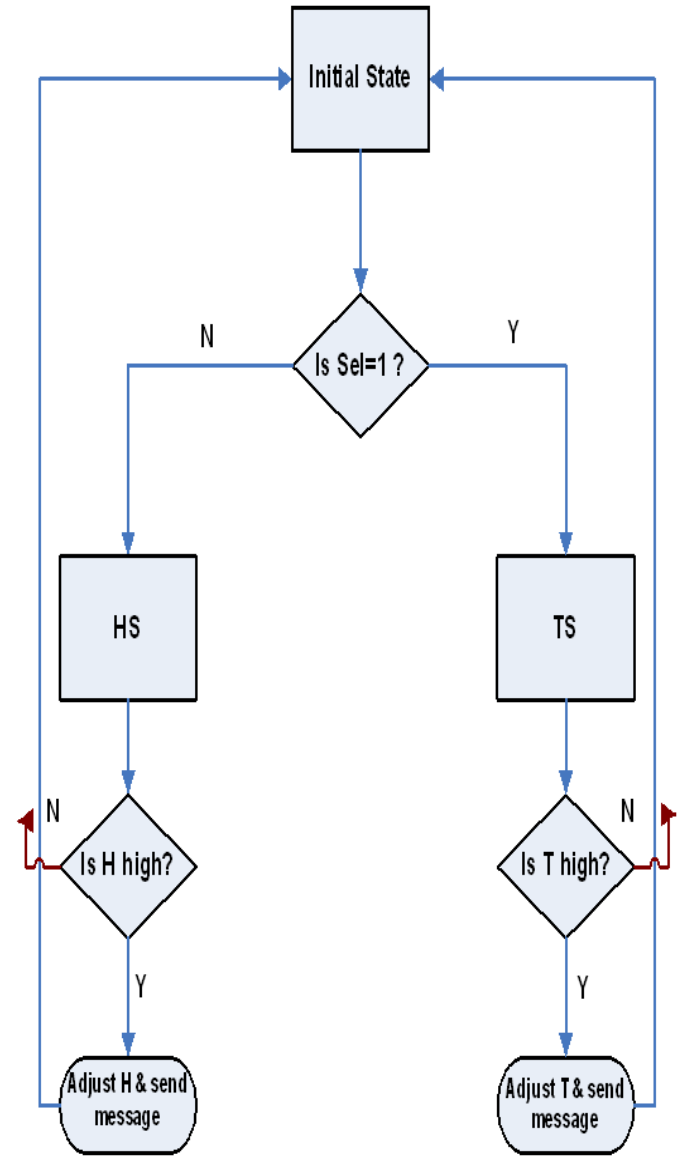

Fig. 3 - Simplified SM Chart for the sensing process 


\section{SIMULATION RESULTS}

The VHDL models have been simulated functionally to verify the correctness of the behavioral description for the models. Fig. 4 shows the simulation results for the temperature sensing and control. Where "clk" is the system clock input, "TS" is an input bit vector of 8-bit which represents the inputs from the temperature sensor. The analog value of the temperature sensor has to be converted to digital value in binary representation using the ADC; this binary value is represented by "TS" input. The signals "SSCATH" is the cathode output for the seven segment display on the system board, and "AN" is the anode output for the seven segment, it is four bits that representing four digits, but only two of them are used. The values on "AN" represent the multiplexing between the seven segment digits, and the values on "SSCATH" represent the seven segment code for the decimal value. The signal "LSD" and "MSD" are internal signal that representing the two BCD (Binary Coded Decimal) digits of the temperature reading. Where "LSD" is least significant digit and "MSD" is most significant digit. Signal "T" is an output signal that indicates the high temperature and normal temperature. In fig. 5 the simulation results for the humidity sensing and control is given, where "PSEN" is reading value for humidity, "SHP" is the output signal that will high for high humidity, "S1" and "C" are the cathode and anode output for the seven segment respectively. Fig. 6 shows the simulation for the multiplexing between the humidity and temperature circuits. Where "TSC" and "HSC" are two 8-bit inputs from the analog to digital converter of the Temperature Sensing Circuit and Humidity Sensing Circuit, "Sel" is the selector input, and "Q" is an 8-bit output of the multiplexer. If (Sel=' 0 ') then $(\mathrm{Q}=\mathrm{HSC})$, and if (Sel='1') then ( $\mathrm{Q}=\mathrm{TSC}$ ). The selector "Sel” is changing sequentially using binary counter. The MSB (Most Significant Bit) of TSC, HSC, and Q are on the left, whether the LSB (Least Significant Bit) are on the right.

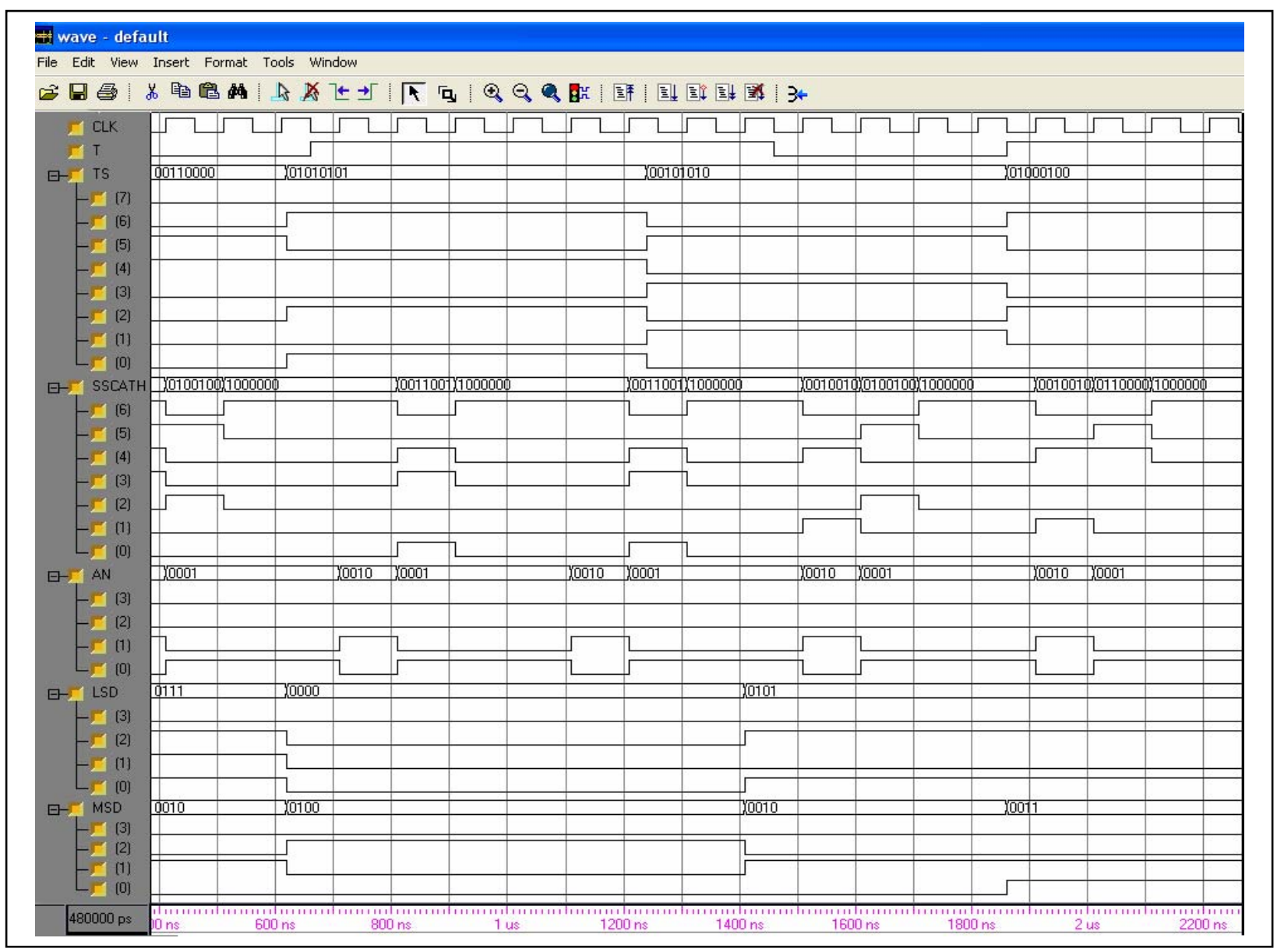

Fig. 4 - Simulation results for the temperature sensing and control 


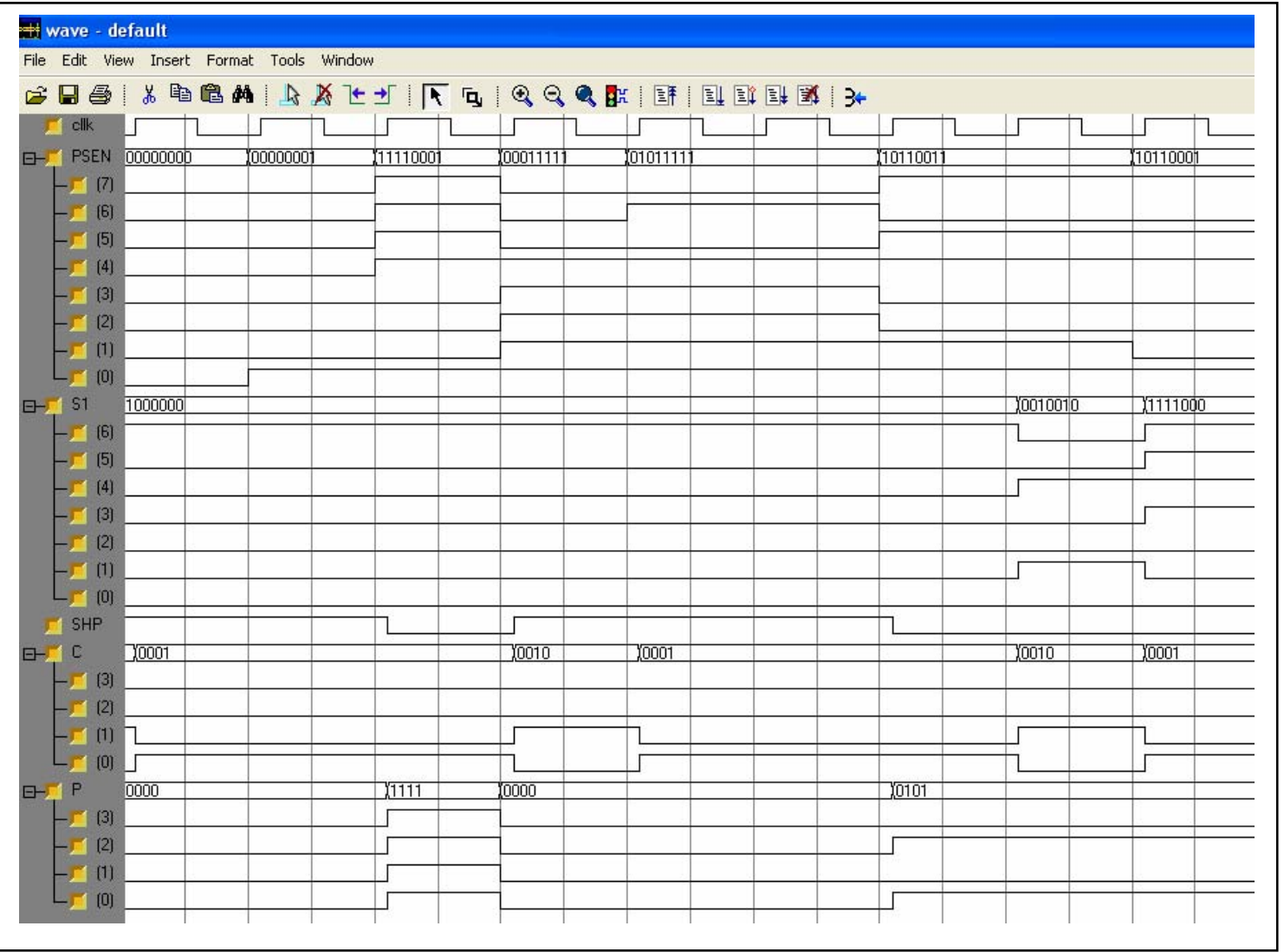

Fig. 4 - Simulation results for the humidity sensing and control unit

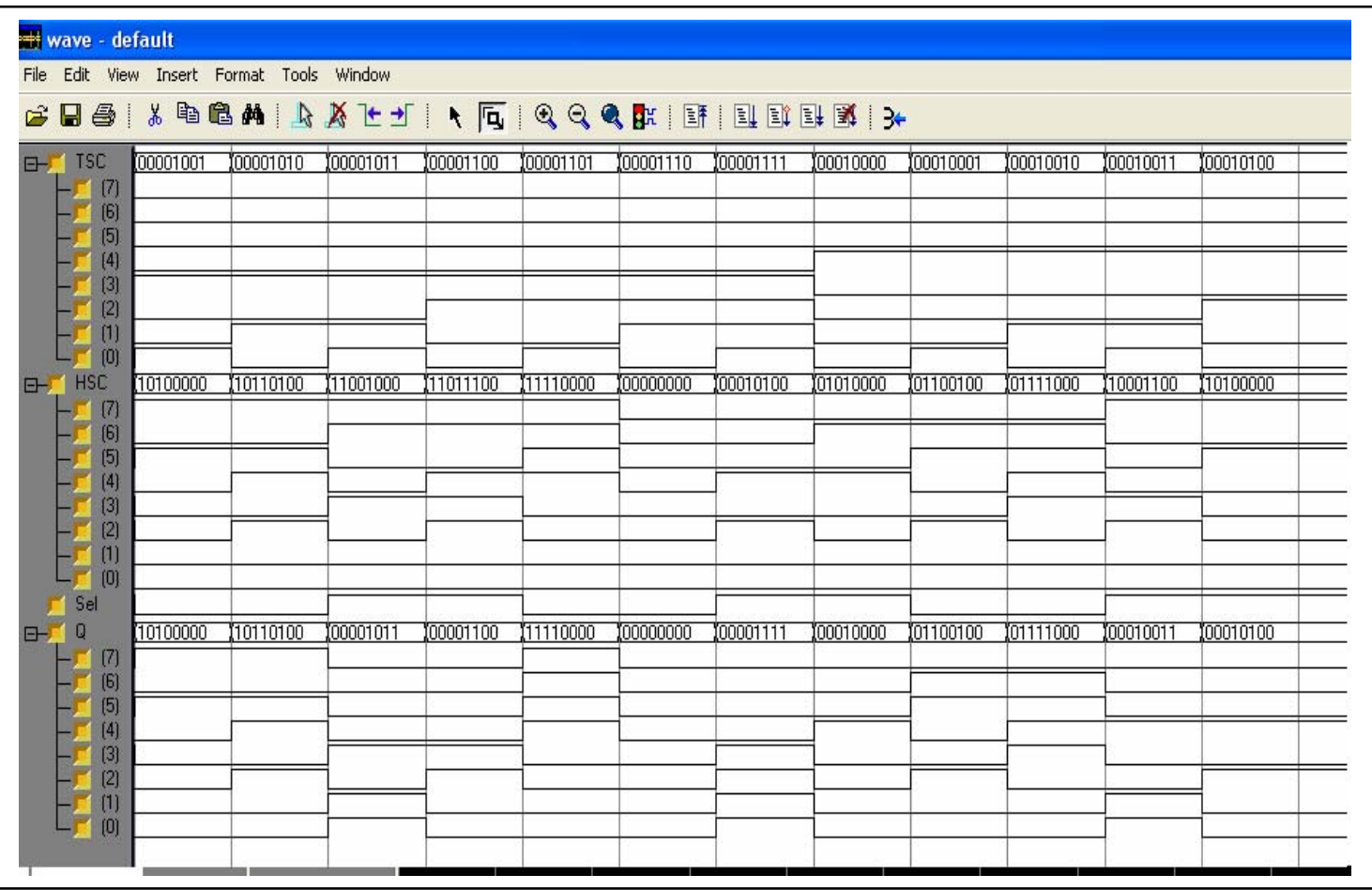

Fig. 5 - Simulation results for the multiplexing between temperature and humidity 


\section{CONCLUSION}

A hardware design of a remote sensing system for humidity and temperature based on using GPRS for internet connection has been proposed in this article. The system was designed using VHDL in a high level design method. The system can remotely sense and control the temperature and humidity through the internet and GPRS network. All parts of the design have been tested in both simulation and hardware level. The implemented design targeted Xilinx Coolrunner CPLD CMOD with 40 pins for final prototype.

\section{ACKNOWLEDGMENT}

The author would like to thank Bader S Abdulla Taqi, Khalifa K Al-Temimi, and Khalifa A Rashed from Computer Engineering Department, IT College, University Of Bahrain for their valuable help.

\section{REFERENCES}

[1] Wayne Wolf, FPGA-Based System Design, Prentice Hall, 2005.

[2] Jan M. Rabaey, Digital Integrated Circuits, A Design Perspective, Second Ed., Prentice Hall, 2003.

[3] Design of a VLSI Integrated Circuit, IEEE, Piscataway, USA.

[4] Chen Xi, Chang Jinzhao, Liu Junfeng, "The monitored control design of the central airconditioning system”, In Control \& Measurment 2007, vol.6, no.1, pp. 245-7.

[5] Jung-Ho Huh, M.J. Brandemuehl, "Optimization of air-conditioning system operating strategies for hot and humid climates” In Energy \& Buildings 2008, vol.40, no.7, pp. 1202-13.

[6] Wu Ming-fang; Tang De-dong, "Smart instrument for measuring petroleum product's humidity based on electromagnetism oscillation technique", In Instrument Techniques and Sensor 2008, no.4, pp. 16-18.

[7] Ji Juan-zao, Shi Jun-yong, Zhang Ji-guang, "Applied controller of temperature and humidity by single-chip microcomputer”, In Instrument Techniques and Sensor 2004, no.10, pp. 10-12.

[8] Li Xin, Qu Meng-ke, Rong Yuz, "Design of temperature and humidity fuzzy controller based on MSP430 MCU:, In Chinese Journal of Sensors and Actuators 2007, vol.20, no.4, pp. 805-8.

[9] W M El-Medany, "FPGA Implementation for Humidity and Temperature Remote Sensing
System”, 14th Annual IEEE International Mixed- Signals, Sensors, and Systems Test Workshop, IMS3TW 08, Canada.

[10] J. M. Jasso, G. O. Vargas, R. C. Miranda, E. V. Ramos, A. Z. Garrido, G. H. Ruiz, "FPGAbased real-time remote Monitoring system", Journal of Computers and Electronics in Agriculture, V 49, 2005, p 272-285.

[11] R C Miranda, E V Ramos, R P Vera, G H Ruiz, "Fuzzy Greenhouse Climate Control System based on a Field Programmable Gate Array", Biosystems Engineering, 2006, 94 (2), pp.165177.

[12] GSM Based Remote Sensing and Control Systems Using FPGA, Wael M El-Medany, Mahmoud R El-Sabry, The IEEE International Conference on Computer \& Communication Engineering, ICCCE’08, Kuala Lumpur, Malaysia, May 2008.

[13] G. Aranguren, L. Nozal, A. Blazquez, and J. Arias, "Remote control of sensors and actuators by GSM", IEEE 28th Annual Conference of the Industrial Electronics Society IECON 02, vol. 3, 2002 p 2306-2310.

[14] Wu, Bing-Fei, Peng, Hsin-Yuan; Chen, ChaoJung "A practical home security system via mobile phones", WSEAS Transactions on Communications, v. 5, 2006, p. 1061-1066.

[15] Eddie M.C. Wong, "A Phone-Based Remote Controller for Home and Office Automation”. IEEE Transactions on Consumer Electronics, Vo1.40, No.1, February 1994, pages 28-34.

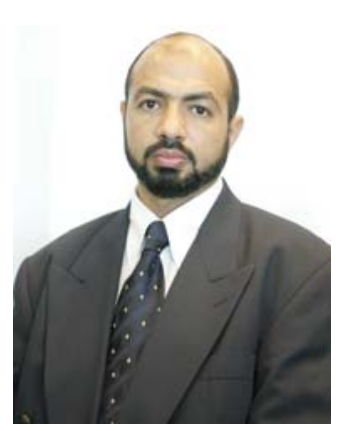

Dr. Wael Mohamed ElMedany is an assistant professor at the department of Communications and Electrical Engineering, Fayoum University, Egypt. Currently he teaches at the department of Computer Engineering, University Of Bahrain. He is IEEE and IEEE Communications Society member. He holds a PhD degree from Manchester University, UK, June 1999, with a major in Electrical Engineering and a minor in Chip Design and its application in error control coding. He got his MSc degree in computer communications from Faculty of Electronic Engineering, Menoufia University, October 1991, and his BSc degree in Electronic Eng from the same University May 1987. El-Medany also held teaching positions in Menoufia University, Cairo University, Obour Academy, Manchester University. His research interest in programmable ASIC design using VHDL. 